\title{
Diagnóstico y tratamiento del trastorno bipolar en población transgénero
}

Inés Arístegui ${ }^{1}$

\author{
Artículo \\ Material original autorizado para la publicación en la revista Psicodebate. \\ Facultad de Ciencias Sociales. Universidad de Palermo. \\ Recibido 19-03-2014 | Aceptado 09-05-2014
}

\section{Resumen}

A medida que se supera la idea de que personas transgénero sufren de una patología con los nuevos lineamientos del DSM-5 (la 5ta edición del Manual Diagnóstico y Estadístico de los Trastornos Mentales) y se alcanza el reconocimiento de sus derechos en un marco legal como la Ley de Identidad de Género en Argentina, se espera que las personas transgénero se acerquen a los servicios de salud en general y de atención psicológica en particular. Este artículo presenta una revisión de las investigaciones existentes sobre la prevalencia, el diagnóstico y el tratamiento del trastorno bipolar en población transgénero. Se realiza un recorrido por las nuevas definiciones del DSM-5, se analizan las dificultades y se presentan consideraciones para su diagnóstico y tratamiento. Debido a los altos índices de depresión, intentos de suicidios y abuso de sustancias presentes en esta población, se estima que existe una alta prevalencia del trastorno bipolar, pero que esta enfermedad no es debidamente diagnosticada ya sea porque las personas transgénero no acceden a los servicios de salud o bien porque algunos de sus síntomas se confunden con las características de los estilos de vida que muchas personas llevan. De la revisión bibliográfica se desprende el requisito de realizar estudios locales para conocer las características de la población transgénero en Argentina, así como la necesidad de capacitar y sensibilizar a los profesionales de la salud mental sobre las problemáticas específicas de estas poblaciones.

Palabras Clave: Trastorno Bipolar - Transgénero - DSM5 - Identidad de Género - Diagnóstico y Tratamiento.

1 Universidad de Palermo - Fundación Huésped, ines.aristegui@huesped.org.ar 


\section{Abstract}

As the idea that transgender people are individuals who suffer from a pathology is overcome with the new guidelines of the DSM-5 and the recognition of their rights with a legal framework such as the Gender Identity Law in Argentina, it is expected that this population will begin to approach health care services in general and mental health aid in particular. This article reviews what is known about the prevalence, diagnosis and treatment of bipolar disorder in transgender people. Firstly, new definitions of DSM-5 are introduced; next, studies on prevalence of psychopathology in this population are reviewed; and finally, challenges and considerations for the diagnosis and treatment of bipolar disorder are presented. Due to the high rates of depression, suicide attempts and substance abuse, it is likely that the prevalence of bipolar disorder is high in this group but is not appropriately diagnosed. This might be due to the lack of access to the health care system or due to the probable confusion between symptoms of the disorder and the lifestyle characteristics of this population. It is concluded that there is a need of conducting local studies to explore the profile and characteristics of this population as well as the need of providing training on transgender specific issues to professionals in the field of mental health.

Keywords: Bipolar Disorder - Transgender - DSM 5 - Gender Identity Diagnosis and Treatment. 
La literatura científica sobre la atención psicoterapéutica de personas lesbianas, gay, bisexuales y transgénero (LGBT) es cada vez mayor y cada vez son más las organizaciones de profesionales que se han pronunciado o elaboran guías de buenas prácticas para trabajar con estas poblaciones (por ejemplo, la Asociación de Psicólogos Americana -APA- y la Sociedad de Psicología de Australia APS-, entre otros). Sin embargo, son pocos los estudios en relación a la atención, prevalencia y comorbilidad de enfermedades mentales graves en esta población (Lucksted, 2004), y aún más escasos aquellos que describen la situación particular de las personas transgénero (Cole, O’Boyle, Emory, \& Meyer, 1997; Mizock \& Fleming, 2011). Históricamente, el vínculo de esta población y los equipos de salud mental se limitó a la aprobación de un estado de aptitud, mediante un diagnóstico de trastorno de identidad de género o disforia de género, para acceder a un tratamiento hormonal o a una intervención quirúrgica que permita adecuar sus cuerpos a la identidad percibida o cambiar sus nombres en los documentos nacionales de identidad (Helien \& Piotto, 2012).

A medida que se avanza en dejar de considerar a las personas transgénero como individuos que padecen una enfermedad mental en sí misma con la 5ta edición del Manual Diagnóstico y Estadístico de los Trastornos Mentales (DSM5, American Psychiatric Association, 2013), y se logran cambios socio-políticos que promueven sus derechos y la reducción del estigma a partir de un marco legal como la Ley de Identidad de Género, se espera que esta población salga del ostracismo y se acerque a los servicios de salud en general y a la atención psicológica en particular (APA, 2008; 2009; Bockting, Knudson \& Goldberg, 2006; DSM-5, 2013). De este modo, cobra mayor relevancia la necesidad de conocer mejor el perfil de este grupo poblacional. Dado que la prevención, la detección temprana y el adecuado tratamiento son aspectos fundamentales en la atención de enfermedades psiquiátricas crónicas, el presente trabajo tiene como objeto explorar diferentes aspectos y consideraciones para el diagnóstico y el tratamiento del trastorno bipolar en población transgénero. A tal fin, primero se realizará un breve recorrido sobre las características de la población transgénero; y seguidamente se propondrán una serie de pautas a considerar para el diagnóstico y tratamiento del trastorno bipolar.

\section{Definiendo a la población transgénero}

Según la APA (2009), el término transgénero es un concepto abarcativo que se utiliza para describir a todas aquellas personas cuya identidad de género no se corresponde con la división tradicional de género femenino y masculino y difiere del sexo asignado al nacer. Este término incluye a las personas transexuales, 
travestis e intersex, entre otros. Según esta entidad, para poder trabajar con personas no conformes con su género, es necesario comprender la distinción entre el sexo biológico, que incluye atributos físicos como cromosomas, gónadas, estructura reproductiva interna y genitales externos; la identidad de género, que refiere a la percepción psicológica de sentirse hombre o mujer y está asociada a la forma en la que las personas se comportan, interactúan y sienten acerca de sí mismas; y los roles de género, definidos como la serie de comportamientos, actitudes y rasgos de personalidad que una sociedad, en un momento histórico determinado, designa como masculinos o femeninos (es decir, propios de los hombres o de las mujeres).

El DSM-5 (2013) publicado recientemente establece que si bien considera que muchas personas transgénero tienen sentimientos de malestar debido a la no congruencia entre su identidad y el género asignado al nacer, no experimentan necesariamente un sufrimiento tan profundo a causa de esta condición como para ser considerado una patología. Así, en el nuevo DSM, se reemplaza el diagnóstico de Trastorno de Identidad de Género por el de Disforia de Género, manifestando que el aspecto crítico para su diagnóstico es la presencia de niveles de estrés clínicamente significativos que dificulten el normal funcionamiento de la persona. La incongruencia entre el sexo biológico y la identidad de género asignada al nacer es un fenómeno poco frecuente y no existen estadísticas precisas sobre su prevalencia. Según un estudio de los Países Bajos, la transexualidad es de 1 en 10,000 para mujeres transgénero (personas que asumen una identidad femenina) y de 1 en 30,000 para hombres transgénero (aquellas que asumen una identidad masculina) (Bakker, van Kesteren, Gooren, \& Bezemer, 1993).

\section{Evaluaciones psicodiagnósticas en población transgénero}

La Asociación Mundial de Profesionales para la Salud Transgénero (World Professional Association of Transgender Health, WPATH) sugiere que los psicodiagnósticos se conviertan en herramientas útiles y necesarias para orientar a los profesionales de la salud acerca de la existencia o ausencia de trastornos psiquiátricos que puedan dificultar el tratamiento o la adaptación que deben alcanzar estos pacientes tanto antes como después de los tratamientos de hormonización o las intervenciones de reasignación de sexo (Feldman \& Goldberg, 2006; WPATH, 2011).

Otras demandas frecuentes de evaluaciones psicodiagnósticas, ya no relacionadas a las intervenciones biomédicas para adecuación del cuerpo a su identidad, surgen por la preocupación de los padres y/o docentes ante el comportamiento de algunos niños que presentan conductas, preferencias y 
características de personalidad que no son típicas para su género (APA, 2009). Por lo general, estos niños tienen dificultades para interactuar con sus pares debido a las conductas de género cruzado y tienden a aislarse socialmente. Asimismo, algunos psicodiagnósticos son solicitados por otros profesionales de la salud, debido a las fallas en la adherencia en tratamientos médicos; o por juzgados de familia, ante la necesidad de evaluar la aptitud y actitud de personas trans para retener o solicitar la custodia de sus hijos y/o menores a cargo (Bockting et al., 2006; Helien \& Piotto, 2012).

En mayo de 2012 se aprobó en Argentina la Ley Nro. 26.743 de Identidad de Género (Boletín Oficial, 2012) que garantiza el libre desarrollo de las personas conforme a su identidad de género, corresponda o no con el sexo asignado al momento de nacimiento. Esta ley, no sólo garantiza el cambio de nombre en sus documentos de identidad, sino también el acceso a una salud integral, tratamientos hormonales e intervenciones quirúrgicas parciales o totales sin requerir autorización judicial o administrativa, sino solo con el consentimiento informado de la persona. A diferencia de otros países con leyes similares, en Argentina deja de ser un requisito la evaluación psicodiagnóstica previa a cualquiera de estas instancias.

\section{Psicopatología en población transgénero}

Aunque son pocos los estudios existentes, se ha observado que la mayoría de las personas transgénero no presentan trastornos psiquiátricos (Bockting et al., 2006; Gómez-Gil, Vidal-Hagemeijer, \& Salamero, 2008). Al estudiar la prevalencia de enfermedades mentales severas, definiéndolas como una disrupción en el ánimo o la personalidad que afecta la vida, el trabajo o las relaciones de las personas, no se han encontrado frecuencias más altas en población transgénero que acude a clínicas especializadas en comparación con la población general (Cole et al., 1997). Sin embargo, otros estudios muestran una mayor incidencia de trastornos de personalidad severos, psicosis y otras enfermedades mentales en muestras clínicas de personas transgénero cuando se la compara con otras poblaciones clínicas (Bodlund, Kullgren, Sundbom, \& Höjerback, 1993; Hartmann, Becker, \& Rueffer-Hesse, 1997). En particular, se observan mayores niveles ansiedad, depresión, abuso de sustancias e intentos de suicidio que en la población en general (Bockting, Huang, Ding, Robinson \& Rosser, 2005; Clements-Nolle, Marx, Guzman, \& Katz, 2001).

Hasta principios de la década de los '80, varios autores consideraban que la transexualidad era un subtipo del espectro de los trastornos límites de personalidad (Murray, 1985; Pearson \& Ovesey, 1974). Bajo el diagnóstico de bordeline podía explicarse la discrepancia que algunos profesionales encontraban 
en la práctica (Lothstein, 1984): si bien las personas transexuales no presentaban ninguna psicopatología en las entrevistas clínicas, exhibían niveles de agresión intenso, falta de integración de la identidad, proceso de diferenciación y límites inadecuados, entre otros.

Actualmente, se estima que muchos de estos trastornos están en relación con el estrés típico de las minorías y la situación de vulnerabilidad en la que viven estas personas, y no debido a su condición de mujer u hombre transgénero en sí misma (APA, 2008, 2009; Feldman \& Goldberg, 2006). Las personas transgénero se han caracterizado por presentar altos niveles de estrés emocional como resultado de las experiencias de estigma y discriminación vividas, y a una seria de múltiples estresores, como ser el rechazo familiar y social, el desempleo, la pobreza y la dificultad para que su identidad sea reconocida (Gómez-Gil et al., 2008). Para muchas personas, la falta de acceso a tratamientos de hormonización o cirugías para adecuar su cuerpo a su identidad de género es una fuente importante de estrés y malestar que puede disminuir al acceder a los procedimientos deseados (Madeddu, Prunas, \& Hartmann, 2009). En particular, Hepp, Kraemer, Schnyder, Miller, y Delsignore (2005) han observado que la prevalencia de trastornos de identidad de género (utilizando el DSM-IV-R) en comorbilidad con trastornos del ánimo es de aproximadamente del $45 \%$ cuando ésta se evalúa a lo largo de la vida y no sólo la comorbilidad al momento del estudio.

Gómez-Gil y sus colegas (2008) encontraron que las mujeres transgénero presentan niveles mayores de depresión que las mujeres heterosexuales. En la misma línea, cuando se comparó las muestra clínicas de la población LGBT (en base a su sexo biológico) con controles de pacientes clínicos que no pertenecieran a la comunidad LGBT, se observó que aquellos cuyo sexo biológico era varón, presentaban menor frecuencia de trastornos psicóticos (excepto los trastornos esquizoafectivos) y mayor frecuencia de trastornos del ánimo que los hombres (Hellman, Sudderth, \& Avery, 2002). Por tanto, es posible hipotetizar que la prevalencia de trastornos del ánimo en mujeres transgénero será considerablemente más alto que en la población general. En particular, algunos autores han planteado causas biológicas para la asociación entre la transexualidad y la bipolaridad (Case \& Ramachandran, 2012). En un estudio con pacientes transgénero, denominados bigénero debido al continuo viraje de género, ha encontrado que la muestra también presenta una tasa elevada de trastorno bipolar.

\section{Diagnóstico de trastorno bipolar}

El Trastorno Bipolar (TB) es una enfermedad psiquiátrica crónica, caracterizada por una marcada inestabilidad en el estado de ánimo, con períodos 
de humor expansivo que se alternan con períodos de humor depresivo (DSM-5, 2013). Como Lolich, Vázquez y Leiderman (2010) sostienen, el diagnóstico de trastorno bipolar (TB) es generalmente tardío, debido a que el mismo se realiza en base a la presencia de un episodio maníaco (TB de Tipo I) o hipomaníaco (TB de Tipo II). Según estos autores, en Argentina el retraso en el diagnóstico es de 8 años aproximadamente ya que la mayoría de las personas comienzan con un episodio de depresión unipolar. Considerando que los índices de depresión en población transgénero oscilan entre el 52\% y el 62\% (Bockting et al., 2005; Clements-Nolle et al., 2001), es posible pensar que una proporción de personas transgénero están erróneamente diagnosticadas. El desafío adicional que presenta esta población para poder diagnosticar adecuadamente el TB, es la falta de permanencia en el sistema de salud debido a las situaciones de estigma y discriminación por parte de los efectores de salud, o el acceso tardío al mismo ante la presencia de otras enfermedades que establecen diferentes prioridades de atención (Hellman \& Klein, 2004; Mizock \& Lewis, 2008).

Si bien la mayoría de las personas que tienen un episodio maníaco no presentan síntomas psicóticos (Lolich et al., 2010), algunos autores han manifestado dificultad para distinguir entre transexualismo y los deseos transexuales inducidos por una psicosis bipolar. En algunos casos de estudio se observó que los pacientes manifestaban características transexuales como deseos de hormonización y operaciones de reasignación de sexo, sólo durante un episodio maníaco y disminuían su comportamiento transexual luego de ser medicados para la manía (Habermeyer, Kamps, \& Kawohl, 2003; Meyer \& Kapfhammer, 1995). En la misma línea, al estudiar pacientes con esquizofrenia durante un periodo psicótico (Baltieri \& De Andrade, 2009), se observó que luego de recibir un tratamiento antipsicótico adecuado, el comportamiento de género diferente al asignado al momento de nacer persistía, pero no así sus deseos de realizar una intervención quirúrgica de sus genitales. Es importante tener en cuenta que este tipo de perspectivas ha llevado a etiquetar a la identidad transgénero como una creencia delirante que no debe ser reforzada ni reconocida por los médicos/personal de salud (Mizock \& Fleming, 2011). Si bien son pocos los casos reportados de co-ocurrencia de episodios maníaco-psicóticos y variaciones en la identidad de género, la prevalencia de esta presentación clínica es difícil de medir considerando las barreras que encuentran las personas transgénero para acercarse a los servicios de salud (Hellman \& Klein, 2004).

Otro aspecto a considerar durante el diagnóstico es la similitud y posible confusión de varios de los criterios diagnósticos para el episodio hipo/maníaco del DSM-5 (2013) -como ser la autoestima exagerada, la disminución de la necesidad de dormir, la atención dispersa, y el exceso de indiscreciones 
sexuales- con ciertas características propias de las condiciones de vida de muchas personas transgénero - por ejemplo, trabajo sexual, consumo de sustancias, infecciones de transmisión sexual sin tratamiento, estrategias de afrontamiento de tipo confrontativa y estilo de comunicación defensivo. Como se describe en el DSM-5 (2013), algunos individuos durante sus fases maníacas presentan un tipo de conducta antisocial, mostrándose hostiles y amenazantes físicamente, como también pueden presentarse con un comportamiento y una apariencia sexualmente sugestivos. Así, es importante para el diagnóstico apropiado de TP, que el profesional pueda discernir si estas manifestaciones se deben a un episodio maníaco o a la forma en que muchas personas trans se acercan a los sistemas de salud como forma de afrontar el estigma y la discriminación (Hellman \& Klein, 2004; Mizock \& Lewis, 2008).

Algunos autores sugieren, el diagnóstico de TB podría mejorarse si se cuenta con la presencia de un miembro de la familia durante la evaluación inicial, o se aplica una escala hetero-administrada (Hirschfeld et al., 2000) como la Escala Diagnóstica del Espectro Bipolar (Vázquez, Romero, Fabregues, Pies, Ghaemi, $\&$ Mota-Castillo, 2010). Esto es importante, ya que difícilmente el paciente considere un episodio hipomaníaco o maníaco como síntomas de malestar y por tanto es necesario contar con alguien que pueda referir a distintas etapas de la vida de esa persona. Sin embargo, este tipo de evaluación es más difícil en las personas transgénero dado que generalmente, son expulsadas de sus hogares y obligadas a migrar a otras ciudades desde muy temprana edad (Helien \& Piotto, 2012). Debido a la migración y a sus condiciones de vida no siempre cuentan con redes de apoyo social estable. De este modo, podría ser útil para hacer un diagnóstico más preciso considerar la presencia de alguna otra persona transgénero con quien el paciente tenga una relación estrecha, a fin de realizar la evaluación.

\section{Tratamiento del trastorno bipolar}

A través de estudios longitudinales, se ha observado que la mayoría de los pacientes logran la remisión de los síntomas agudos cuando están bajo tratamiento; sin embargo, sólo una pequeña proporción recupera su funcionalidad de forma equiparable (Tohen et al., 2010). Según Barrera, Vázquez, Tannenhaus, Lolich y Herbst (2012), se observa una merma en la funcionalidad en áreas como autonomía, finanzas, ocio y funcionamiento interpersonal y cognitivo. Asimismo, la calidad de vida de los pacientes con trastorno bipolar se ve afectada por los altos niveles de estigmatización, teniendo un efecto negativo sobre la autoestima de la persona (Mileva, Vázquez, \& Milev, 2013). En particular, las personas transgénero con diagnóstico de TB estarían frente a un potencial doble de estigmatización 
que podría incrementar los niveles de ansiedad y como consecuencia dificultar la adherencia al tratamiento y por tanto disminuir aún más sus niveles de funcionalidad. Establecer la merma en el nivel de funcionalidad de las personas transgénero como consecuencia del TB no sería sencillo considerando que estas personas presentan bajo nivel educativo, desempleo e aislamiento social, y déficits cognitivos resultantes del abuso de sustancias y/o infecciones de transmisión sexual sin tratamiento como sífilis y VIH.

Aunque cada vez cobra más relevancia el componente genético heredable y las alteraciones neurobiológicas en relación a la etiología del TB, los factores psicosociales pueden actuar como factores protectores, o en caso contrario, precipitar la aparición de las alteraciones del ánimo o incrementar la frecuencia y velocidad del viraje. Esto realza la importancia de considerar un tratamiento integral cuando se trabaja con población transgénero, ya que los factores psicosociales más que operar como factores protectores podrían acelerar la aparición de nuevos episodios activos.

En relación a la medicación, Lolich et al. (2010) señalan que si el paciente presenta psicosis en su primer episodio, al recibir el diagnóstico más temprano y por tanto la medicación adecuada, podrá presentar mejores niveles de funcionalidad. Sin embargo, como la mayoría de los TB de tipo II debutan con depresión, son mal diagnosticados y por tanto reciben un tratamiento erróneo con antidepresivos que puede conducir a virajes (Tondo, Vázquez, \& Baldessarini, 2010). Lamentablemente, por lo general las personas transgénero llegan tardíamente a los servicios de salud y no presentan una buena adherencia a los tratamientos crónicos que demandan regularidad de consultas y toma de medicación debido a las dificultades para organizar sus horarios de trabajo nocturno con la atención hospitalaria por las mañanas, el miedo a experimentar situaciones de estigma y discriminación, los estados de pérdida de consciencia por el consumo de sustancias y la falta de interés en general.

La falta de un tratamiento farmacológico adecuado, podría entonces incrementar el riesgo de suicidio que ya de por sí es 15 veces más alto para las personas con trastorno bipolar que para la población general (DSM-5, 2013), y en caso de las personas transgénero en particular, varios estudios han registrado una prevalencia de 37\% de intentos reportados de suicidios (APA, 2009; Cole et al., 1997; Mizock \& Fleming, 2011).

Lolich, Vázquez, Álvarez y Tamayo (2012) observaron que la mayoría de las intervenciones psicosociales, más allá de su marco teórico, tiene los mismos objetivos: reducir el número de recaídas, mejorar la calidad de vida y mejorar la comunicación. Algunas terapias, como la interpersonal, promueven un estilo de vida más organizado para reducir el nivel de estresores, otras apuntan a regular 
el sueño y alcanzar un descanso adecuado. Sin embargo, este tipo de objetivos podría ser difícil de logar con una población que mayormente realiza trabajo sexual, lleva una vida nocturna, y está expuesta a altos niveles de violencia en la calle, abuso de drogas y alcohol (Bockting et al., 2006).

Del mismo modo, las terapias de tipo cognitiva o psico-educativas que han demostrado ser muy efectivas en el tratamiento del TB (Lolich et al., 2012), plantean el desafío de solicitar a las personas transgénero la asistencia a un cierto número de sesiones pautadas. Para aplicar este tipo de estrategias con esta población en particular, podrían pensarse dispositivos que combinen varias temáticas, formando así grupos de pares y que coincidan con los días en que asisten a los centros de salud para hacer los controles de sus tratamientos de hormonización, que actualmente son la puerta de entrada de la población trans al sistema de salud.

\section{Conclusión}

El objeto de este trabajo ha sido reflexionar acerca de las particularidades de la población transgénero y cómo estas podrían influir en la prevalencia y el proceso de diagnóstico y tratamiento de enfermedades psiquiátricas como el trastorno bipolar. Como se ha presentado a lo largo de este trabajo, la literatura es escasa y se deriva de investigaciones realizadas en otros países, principalmente América del Norte y Europa. Asimismo, la mayoría de las investigaciones se han realizado con muestras de personas que se acercan a las clínicas para acceder a tratamientos médicos, y por tanto, no son generalizables a la población transgénero en su totalidad.

De la revisión es posible concluir que debido a los altos índices de depresión, intentos de suicidios y abuso de sustancias, es esperable que un gran número de personas transgénero cumplan con los criterios diagnósticos de TB y no estén adecuadamente diagnosticadas. Esto podría deberse a que las personas transgénero no acceden a los servicios de salud o llegan con otras urgencias, o bien porque algunos síntomas de la enfermedad se confunden con características de sus condiciones y estilos de vida y por tanto, se subestimen algunos criterios diagnósticos. Asimismo, es probable que algunas personas transgénero hayan recibido el diagnóstico de depresión unipolar y estén erróneamente tratadas, y por consiguiente, sujetas a provocar un primer episodio hipo/maníaco. Cualquiera sea el caso, el riesgo de suicidios y la co-ocurrencia de abusos de sustancias es muy alto. El tratamiento de la población transgénero con TB podría implicar grandes desafíos debido a las condiciones de vida de las mismas, la falta de acceso y retención en el sistema de salud y el impacto emocional negativo de los altos índices de estigma y discriminación que viven a diario. 
En resumen, existen brechas de conocimiento en relación a la prevalencia, diagnóstico y tratamiento del trastorno bipolar en la población transgénero. Esto se debe no sólo a que se trata de una muestra poblacional relativamente pequeña y difícil de hallar, sino también, a la falta de registro de la orientación sexual y la identidad de género como datos en los estudios epidemiológicos que comúnmente se realizan. Considerando la situación de vulnerabilidad en la que estas comunidades viven en nuestro país, podría pensarse en la necesidad de realizar investigaciones que permitan arrojar luz a varias de las hipótesis que este artículo ha planteado.

Si bien la ley de identidad de género garantiza el acceso a los tratamientos médicos de feminización o readecuación de sexo sin un diagnóstico psiquiátrico, esto no desliga la responsabilidad de los profesionales de la salud de realizar una anamnesis en profundidad a fin de descartar cualquier enfermedad mental que podría tener un efecto negativo sobre sus tratamientos. Es importante para el bienestar de las personas transgénero que los psicólogos que traten con ellas tengan conocimiento especializado sobre sus problemáticas. Los altos niveles de estigma, discriminación y conflictos internos que muchos de estos individuos viven en su cotidianeidad, los expone al riesgo de desarrollar problemas de salud mental, como ser abuso de sustancias, ansiedad y depresión, así como aumentar la frecuencia de episodios y/o incrementar la posibilidad de manifestar enfermedades mentales severas. 


\section{Referencias}

American Psychiatric Association (2013). Diagnostic and statistical manual of mental disorders (5ta ed.). Arlington, VA: American Psychiatric Publishing.

American Psychological Association (2008). Resolution on transgender and gender identity and gender expression non-discrimination. Recuperado el 18 de mayo de 2010 de http://www.apa.org/pi/lgbt/programs/transgender/index.aspx

American Psychological Association (2009). Report of the Task Force on Gender Identity and Gender Variance. Washington, DC: Author.

Bakker, A., van Kesteren, P. J. M., Gooren, L. J. G., \& Bezemer, P. D. (1993). The prevalence of transsexualism in the Netherland. Acta Psychiatrica Scandinavica, 87, 237-238

Baltieri, D. A., \& De Andrade, A. G. (2009). Schizophrenia modifying the expression of gender identity disorder. The Journal of Sexual Medicine, 6(4), 1185-1188.

Barrera, A., Vázquez, G., Tannenhaus, L., Lolich, M., \& Herbst, L. (2013). Teoría de la mente y funcionalidad en pacientes bipolares en remisión sintomática. Revista de Psiquiatría y Salud Mental, 6(2), 67-74.

Bockting, W., Knudson, G., \& Goldberg, J. M. (2006). Counseling and mental health care of transgender adults and loved ones. International Journal of Transgenderism, 9, 35-82.

Bockting, W. O., Huang, C. Y., Ding, H., Robinson, B., \& Rosser, B. R. S. (2005). Are transgender persons at higher risk for HIV than other sexual minorities? A comparison of HIV prevalence and risks. International Journal of Transgenderism, 8(2/3), 123-131.

Bodlund, O., Kullgren, G., Sundbom, E., \& Höjerback, T. (1993). Personality traits and disorders among transsexuals. Acta Psychiatrica Scandinavica, 88, 322-327.

Boletín Oficial de la República Argentina (mayo 2012). Ley de Identidad de Género. Año CXX, Nro. 32.404. Recuperado el 20 de septiembre de 2013 de http://www1.hcdn.gov.ar/BO/boletin12/2012-05/BO24-05-2012leg.pdf

Case, L. K., \& Ramachandran, V. S. (2012). Alternating gender incongruity: a new neuropsychiatric syndrome providing insight into the dynamic plasticity of brain-sex. Medical Hypotheses, 78(5), 626-631. 
Clements-Nolle, K., Marx, R., Guzman, R., \& Katz, M. (2001). HIV prevalence, risk behaviors, health care use, and mental health status of transgendered persons in San Francisco: Implications for public health intervention. American Journal of Public Health, 91(6), 915-921.

Cole, C. M., O’Boyle, M., Emory, L. E., \& Meyer, W. J. (1997). Comorbidity of gender dysphoria and other major psychiatric diagnoses. Archives of Sexual Behavior, 26, 13-26.

Feldman, J. L., \& Goldberg, J. M. (2006). Transgender primary medical care. International Journal of Transgenderism, 9, 3-34.

Gómez-Gil, E., Vidal-Hagemeijer, A., \& Salamero, M. (2008). MMPI-2 characteristics of transsexuals requesting sex reassignment: Comparison of patients in prehormonal and presurgical phases. Journal of Personality Assessment, 90(4), 368-374.

Habermeyer, E., Kamps, I., \& Kawohl, W. (2003). A case of bipolar psychosis and transsexualism. Psychopathology, 36, 168-1703.

Hartmann, U., Becker, H., \& Rueffer-Hesse, C. (1997) Working with transgendered and transsexual people as offenders in the probation service. Probation Journal, 49, 227-232.

Helien, A. \& Piotto, A. (2012). Cuerpxs equivocadxs: Hacia la comprensión de la diversidad sexual. Buenos Aires: Paidos.

Hellman, R. E., \& Klein, E. (2004). A program for Lesbian, Gay, Bisexual, and Transgender individuals with major mental illness. Journal of Gay \& Lesbian Psychotherapy, 8(3-4), 67-82.

Hellman, R. E., Sudderth, L., \& Avery, A. M. (2002). Major mental illness in a sexual minority psychiatric sample. Journal of the Gay and Lesbian Medical Association, 6, 97-106.

Hepp, U., Kraemer, B., Schnyder, U., Miller, N., \& Delsignore, A. (2005). Psychiatric comorbidity in gender identity disorder. Journal of Psychosomatic Research, 58, 259-2612.

Hirschfeld, R., Spitzer, R., Calabrese, J., Flynn, L., Lewis, L., McElroy, S., ... Zajecka, J. (2000). Development and validation of a screening instruments for bipolar spectrum disorder: the mood disorder questionnaire. American Journal of Psychiatry, 157, 1873-1875. 
Lolich, M., Vázquez, G., \& Leiderman, E. A. (2010). Primer episodio psicótico en trastorno bipolar: Diferenciación clínica e impacto funcional en una muestra argentina. Vertex: Revista Argentina de Psiquiatría, 94, 418-427.

Lolich, M., Vázquez, G., Álvarez, L., \& Tamayo, J. (2012) Psychosocial interventions on bipolar disorders: A review. Actas Psiquiátricas Españolas 40(2), 84-92.

Lothstein, L. M. (1984). Psychological testing with transsexuals: A 30-year study. Journal of Personality Assessment, 48(5), 500-507.

Lucksted, A. (2004). Lesbian, gay, bisexual, and transgender people receiving services in the public mental health system: Raising issues. Journal of Gay \& Lesbian Psychotherapy, 8(3-4), 25-42.

Madeddu, F., Prunas, A., \& Hartmann, D. (2009). Prevalence of Axis II disorders in a sample of clients undertaking psychiatric evaluation for sex reassignment surgery. Psychiatric Quarterly, 80(4), 261-267.

Mayer, C., \& Kapfhammer, H. P. (1995). Coincidence of transsexuality and psychosis. Der Nervenarzt, 66(3), 225-230.

Mileva, V. R., Vázquez, G. H., \& Milev, R. (2013). Effects, experiences, and impact of stigma on patients with bipolar disorder. Neuropsychiatric Disease and Treatment, 9, 31-40.

Mizock, L., \& Lewis, T. K. (2008). Trauma in transgender populations: Risk, resilience, and clinical care. Journal of Emotional Abuse, 8(3), 335-354.

Mizock, L., \& Fleming, M. Z. (2011). Transgender and gender variant populations with mental illness: Implications for clinical care. Professional Psychology: Research and Practice, 42(2), 208-213.

Murray, J. F. (1985). Borderline manifestations in the Rorschachs of male transsexuals. Journal of Personality Assessment, 49(5), 454-466.

Pearson, E., \& Ovesey, L. (1974). The transsexual syndrome in males: Primary transsexualism. American Journal of Psychotherapy, 28, 4-20.

Tohen, M., Hennen, J., Zarate, C. M., Baldessarini, R. J., Strakowski, S. M., Stoll, A. L., \& Cohen, B. M. (2010). Two year syndromal and functional recovery in 219 cases of first episode major affective disorder with psychotic features. American Journal of Psychiatry, 157, 220-228. 
Tondo, L., Vázquez, G., \& Baldessarini, R. J. (2010). Mania associated with antidepressant treatment: Comprehensive meta-analytic review. Acta Psychiatrica Scandinavia, 121(6), 404-414.

Vázquez, G. H., Romero, E., Fabregues, F., Pies, R., Ghaemi, N., \& Mota Castillo, M. (2010). Screening for bipolar disorders in Spanish-speaking populations: Sensitivity and specificity of the Bipolar Spectrum Diagnostic Scale-Spanish Version. Comprehensive Psychiatry, 51(5), 552-556.

World Professional Association of Transgender Health, WPATH (2011). Standards of Care (7ma ed.). Atlanta: Author. 
